\title{
Shape Control Systems for Sendzimir Steel Mills
}

\author{
John V. Ringwood, Senior Member, IEEE
}

\begin{abstract}
This paper deals with the problem of shape (or flatness) control for Sendzimir 20-roll cold-rolling steel mills. Such mills, with a variety of shape actuators, offer the control engineer considerable choice in the configuration and design of a shape control system. In addition, the Sendzimir mill is a reversing mill, with shape measurement devices at either end of the mill, providing a facility for feedforward of incoming shape disturbances. The multivariable problem presented by the mill has a significant singularity problem, which is tackled in this paper using a variety of approaches, motivated by both practical (operational) considerations and formal theoretical procedures. A final challenge lies in the desire to economize on the number of controllers required to cover the mill over its wide range of operating conditions. The paper compares and contrasts various controller possibilities and is intended to provide a "cookbook" of shape control tools and strategies for Sendzimir mills.
\end{abstract}

Index Terms-Eigenfunctions, eigenvalues, metal industry, multivariable systems, robustness, Sendzimir mill, shape control, singular value decomposition.

\section{INTRODUCTION}

$\mathbf{T}$ HE Senzimir mill under consideration is the 20-high mill, with the rolls arranged in a 1-2-3-4 formation, above and below the strip (see Fig. 1). This configuration is used for rolling hard materials such as stainless steel, the large stack providing support and preventing unwanted bending under the high loads involved. Drive is applied to the cluster via the second intermediate rolls. The first intermediate rolls (FIR's) are tapered, their lateral movement affording one means of shape control, with upper and lower FIR's tapered in opposite directions. The other method of shape control is via the segmented backup rolls at the top of the mill. Movement of the "As-U-Roll" (AUR) racks in or out of the mill cause rotation of eccentrics on the top mill back-up roll shafts which create bending of the back-up roll. This bending permeates through the cluster and is attenuated due to the stack rigidity, the stack behaving like a low-pass filter, in spatial terms. Due to the closer proximity of the FIR's to the strip, their influence as a shape control device is considerable. Both sets of shape actuators are driven by hydraulic motors, which operate at a single speed only. The high-order bending, which is achievable in the Sendzimir mill (Z-mill), allows correction of high-order shape defects such as "herringbone" and "quarterbuckle." This is in contrast to simpler mills, such as the four-high mill, which can only support up to second-order bending. Strip shape is measured $2.91 \mathrm{~m}$ downstream of the roll-gap, using an ASEA Stressometer. Since the Sendzimir mill is a reversing mill, shapemeters are placed at both sides of the

Manuscript received December 3, 1997; revised October 29, 1998. Recommended by Associate Editor, R. Takahashi.

The author is with Control Systems Group, School of Electronic Engineering,

Dublin City University, Glasnevin, Dublin 9, Ireland.

Publisher Item Identifier S 1063-6536(00)00784-3. mill. The Stressometer consists of a segmented roll, with 31 individual segments, where four pressure measurements per revolution of this device are provided, causing a four-period-per-revolution sinusoid to be superimposed on the output signal $(40 \mathrm{~Hz}$ at a speed of $10 \mathrm{~m} / \mathrm{s})$. Further noise on the output signal is introduced due to the $2 \mathrm{kHz}$ magnetizing currents used with the pressure sensors.

Approximately 3000 different schedules are available on the mill, rolling various qualities of stainless steel strip in widths ranging from 0.6 to $1.4 \mathrm{~m}$ and gauges of four down to $0.3 \mathrm{~mm}$. Each schedule, which is composed of a number of passes, (two to 12), can be completed without the need for rethreading of the mill, due to the mill's reversing capability. The different passes and schedules required to achieve a given final gauge for different grades and widths of rolled strip involve variations in mill setup, such as roll diameters and strip speed and changes in material characteristics, such as input and output gauges for each pass, strip width, and material hardness. These cause significant (up to $300 \%$ ) changes in the mill model parameters, which point to a possible requirement for a number of controllers to cover the range of plant operation.

The difficulty of shape control, being a truly multivariable control problem, is manifested by the fact that shape, in the vast majority of multiroll mills, is still controlled using manual control actions. Although there are a variety of mechanisms for controlling strip shape (such as selective roll cooling, roll tilting and work-roll bending), the unique method employed in the Sendzimir mill is eccentric position control, which gives considerable variety in the types of roll bending which can be achieved. Such variety and shape control potential results in a challenging control problem.

Although many of the designs presented in this paper are detailed elsewhere in the published literature, the current paper presents a unified view of each methodology and how the specific problems of singularity and multipass performance are addressed by each design. In order to focus on comparative issues, a large degree of brevity is employed in presenting individual controller solutions, with the interested reader referred to other sources. The section on feedforward control has not previously been reported in the literature. The paper concludes with recommendations for choice of a shape control strategy for the mill under consideration.

\section{SENDZIMIR MiLl ModEL}

The mill model is divided into static and dynamic sections. The static model represents the rolling cluster, while the dynamic model deals with effects in the actuators, strip dynamics, and shapemeter. The model has, in total, ten inputs and eight modeled outputs, with all the interaction assumed to take place in the static model. The dynamic sections are considered to be 


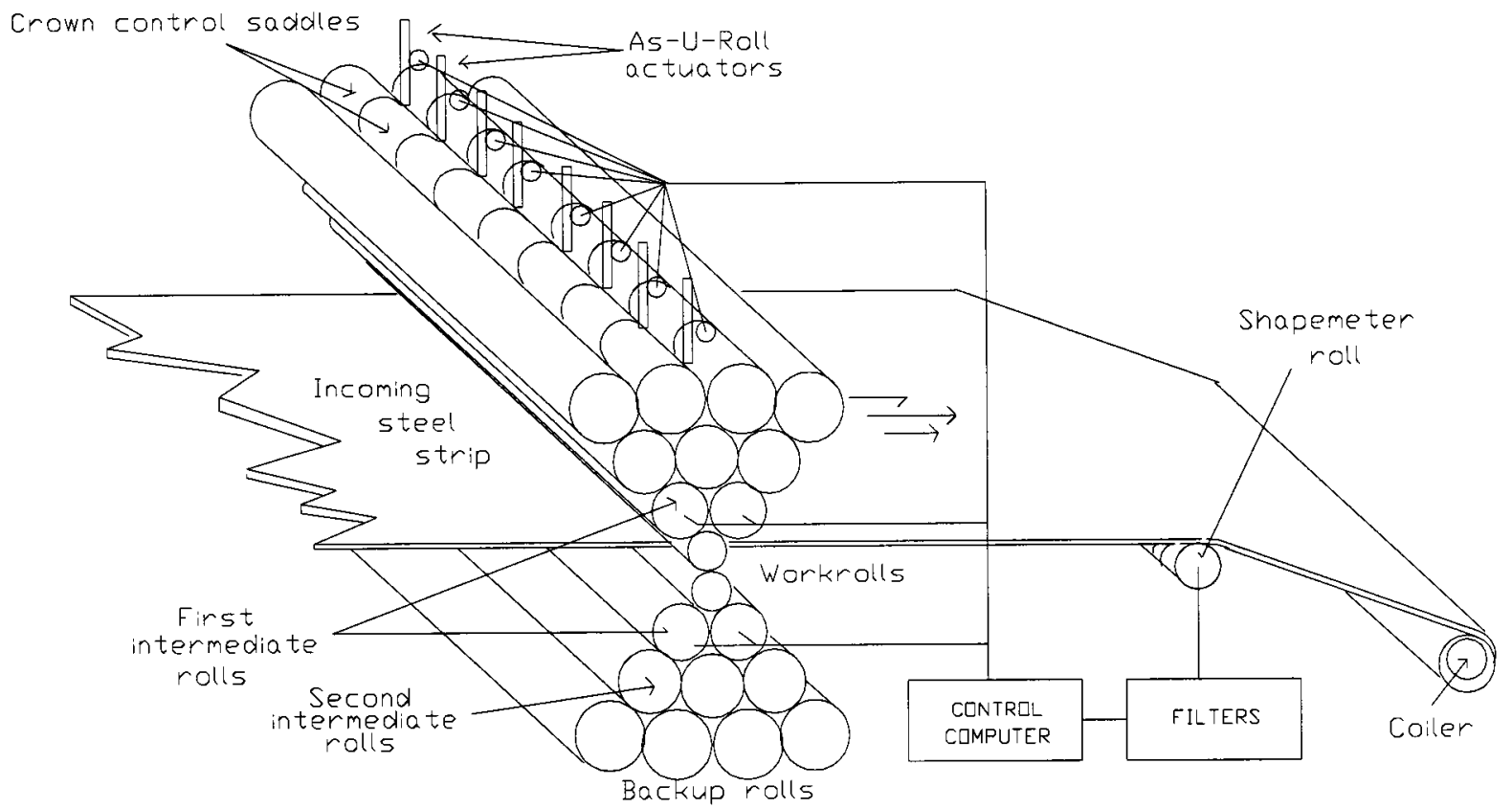

Fig. 1. Physical attributes of Sendzimir mill.

spatially independent, resulting in independent (diagonal) block representations.

\section{A. Static Model}

Since the rolling cluster is under a high compressive load (approx. 5000 tons), changes in shape actuator positions are transmitted immediately to the roll gap. Therefore, the relationship between the shape actuator positions and the roll-gap shape profile is represented by a matrix of constant gains (the mill matrix) as

$$
G_{m}=\left[\begin{array}{ll}
G_{a} & G_{i}
\end{array}\right] \in \mathbb{R}^{8 \times 10}
$$

where

$$
y_{g}=G_{m}\left[\begin{array}{l}
u_{a} \\
u_{i}
\end{array}\right]
$$

where $y_{g} \in \mathbb{R}^{8}$ represents the shape profile at the rollgap, with $u_{a} \in \mathbb{R}^{8}$ and $u_{i} \in \mathbb{R}^{2}$ the Us-U-Roll and intermediate roll actuator positions, respectively. The adoption of a set of linear gains carries with it the assumption of the theorem of superposition, i.e., the net shape effect at the roll-gap is equal to the sum of the individual effects due to AUR and FIR movements separately.

\section{B. Dynamic Model}

Both AUR and FIR actuators are represented by the block diagram in Fig. 2. These present a significant nonlinearity in the system and an obstacle to diagonalization of the system transfer function. Consequently, an actuator linearization technique is outlined in Section II-C, providing the block commutativity property required for diagonalization. The salient features of the actuator subsystems include different parameters for AUR and FIR systems, rate-limited movement and backlash in drive mechanisms. Table I gives the parameters associated with AUR and FIR actuator sets. The strip and shapemeter dynamics vary with the strip speed and are given as

$$
g_{s s}(s)=\frac{e^{-\tau_{1} s}}{\left(1+\tau_{2} s\right)\left(1+\tau_{3} s\right)}
$$

where

$\tau_{1} \quad D / v, \tau_{2}=D_{1} / v$

$D \quad$ Distance from roll-gap to shapemeter $(2.91 \mathrm{~m})$;

$D_{1} \quad$ Distance from roll-gap to coiler $(5.32 \mathrm{~m})$;

$v \quad$ Strip velocity in $\mathrm{m} / \mathrm{s}$;

$\tau_{3} \quad$ Time constant of the shapemeter filter, which is varied for the different strip speeds as in Table II.

The strip dynamics relate to the transport delay between actuation and measurement and the principle of St. Venant [1], which states that the stress variation caused by end traction will decay to zero exponentially due to the difference between input and exit sides of the mill.

\section{Actuator Linearization}

A simple describing function analysis [2] may be used to represent the actuator system in Fig. 2 as

$$
g_{a c t}(s)=\frac{1}{1+\left(\frac{\pi x}{4 k_{c} k_{i}}\right)\left(1-\delta^{2} / x^{2}\right)^{1 / 2} s}
$$

where $x$ is the signal entering the relay. A first-order compensator is now placed in cascade with each actuator of the form

$$
C(s)=\frac{1+\tau s}{1+\tau_{e} s}
$$

with $\tau$ evaluated as the equivalent time constant in (4) and $\tau_{e}$ chosen by the designer, subject to limitations on the max. rate of change of the actuator positions. A value of $\tau_{e}=2.0$ was found to be appropriate. 


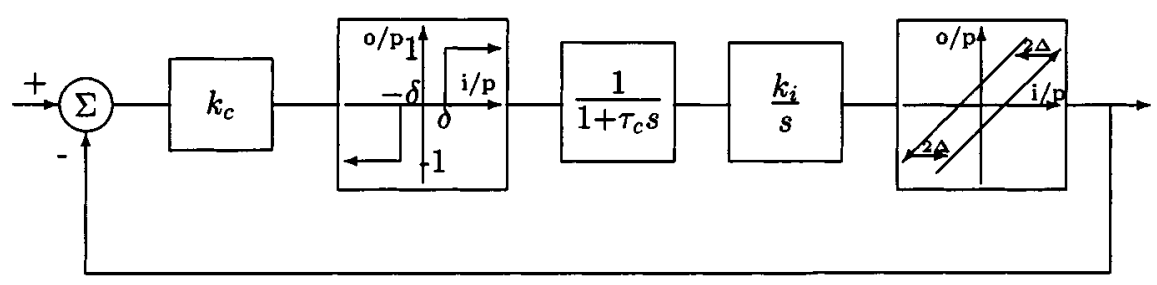

Fig. 2. Block diagram of actuator subsystem.

TABLE I

PARAMETERS FOR AUR AND FIR ACTUATORS

\begin{tabular}{|c|c|c|c|c|c|}
\hline $\begin{array}{c}\text { Actuator } \\
\text { Units }\end{array}$ & $\begin{array}{c}\delta \\
\text { volts }\end{array}$ & $\begin{array}{c}\tau_{c} \\
\text { Secs. }\end{array}$ & $\begin{array}{c}k_{i} \\
\text { gain }\end{array}$ & $\begin{array}{c}\Delta \\
\mathrm{mm}\end{array}$ & $\begin{array}{c}k_{c} \\
\text { gain }\end{array}$ \\
\hline AUR & 0.25 & 0.05 & 8.0 & 0.15 & 1.3 \\
\hline FIR & 0.25 & 0.1 & 3.13 & 0.25 & 0.7 \\
\hline
\end{tabular}

TABLE II VARIATIONS IN SHAPEMETER TIME CONSTANT

\begin{tabular}{|c|c|c|c|}
\hline Strip speed $(\mathrm{m} / \mathrm{s})$ & $0 \rightarrow 2$ & $2 \rightarrow 5$ & $5 \rightarrow 15$ \\
\hline$\tau_{3}$ (secs.) & 1.43 & 0.74 & 0.3 \\
\hline
\end{tabular}

\section{Complete Mill Model}

Following linearization of the actuators, the complete mill model may now be stated as

$$
G(s)=g(s)\left[\begin{array}{ll}
G_{a} & G_{i}
\end{array}\right] \in \mathbb{R}(s)^{8 \times 10}
$$

where

$$
g(s)=\frac{e^{-\tau_{1} s}}{\left(1+\tau_{2} s\right)\left(1+\tau_{3} s\right)\left(1+\tau_{e} s\right)}=\frac{\gamma(s)}{\sigma(s)} .
$$

Features of the mill model which present a challenge to the control engineer include the following.

- The mill matrix, $G_{m}$, suffers from rank deficiency, there being only four reasonably large singular values. A typical SVD spectrum is given as

$$
\begin{array}{r}
\sigma_{i}\left(G_{m}\right)=\left[\begin{array}{cccccc}
9.96 & 7.60 & 4.19 & 1.48 & 0.33 & 0.25 \\
0.091 & 0.025
\end{array}\right], \quad 1 \leq i \leq 8 .
\end{array}
$$

- The mill matrix $G_{m}$ varies with each schedule and pass, as the mill setup and strip parameters change.

- The dynamic section of the system, represented by $g(s)$ varies with mill speed.

- The complete system, including the FIR's, is nonsquare, preventing an attempt at associating particular inputs and outputs. However, with the AUR system alone, such an association is possible by relating actuators and measurements in the same region of the strip.

\section{As-U-Roll Control System Design}

A number of studies have concentrated on an automatic shape control system utilizing the AUR actuators alone. The basis for not including (initially, at least) the FIR's in the automatic control scheme accords with rolling practice, since the FIR's are generally preset for a particular pass, with only the AUR's (as the name suggests) moved while rolling is taking place. Both of the approaches described here address the mill matrix singularity problem by effectively reducing the dimension of the system. Such an approach is suggested by theoretical considerations but is also supported by rolling practice, since no attempt is made by mill operators to control shape profiles higher than fourth order, due, in part at least, to relative positional restrictions on the AUR's in order to prevent fracture of the back-up roll shafts.

\section{A. AUR Design 1-Parameterization Approach}

In this approach, the shape profile is parameterized in terms of a set of coefficients which reflect the components of loworder $(1 \rightarrow 4)$ polynomial profiles. Following a least-squares analysis based on a number of equally spaced measurements [3], [4], the "best" parameter fit is found to be given by the Gram polynomials [5], with the "parameterization" matrix [6] given by (9), shown at the bottom of the page, where

$$
y_{p}=P y
$$

with $y \in R e^{8}$ the measured shape profile (at the shapemeter) and $y_{p} \in \mathbb{R}^{4}$ the parameterized version. The form of these polynomials may be observed in Fig. 3. A more in-depth treatment of such a parameterization, utilizing the Chebyshev polynomials, is given in [7]. The parameterized mill transfer function matrix (TFM), considering only the AUR's as the shape control device, is

$$
G(s)=g(s) P G_{a} P^{T} \in \mathbb{R}(s)^{4 \times 4}
$$

with $g(s)$ as given in (7). From a practical point of view, the application of $P$ and $P^{T}$ respectively parameterize the output

$$
P=\left[\begin{array}{rrrrrrrr}
-0.54 & -0.38 & -0.23 & -0.08 & 0.08 & 0.23 & 0.38 & 0.54 \\
0.54 & 0.08 & -0.23 & -0.38 & -0.38 & -0.23 & 0.08 & 0.54 \\
-0.43 & 0.31 & 0.43 & 0.18 & -0.18 & -0.43 & -0.31 & 0.43 \\
0.28 & -0.53 & -0.12 & 0.36 & 0.36 & -0.12 & -0.53 & 0.28
\end{array}\right]
$$




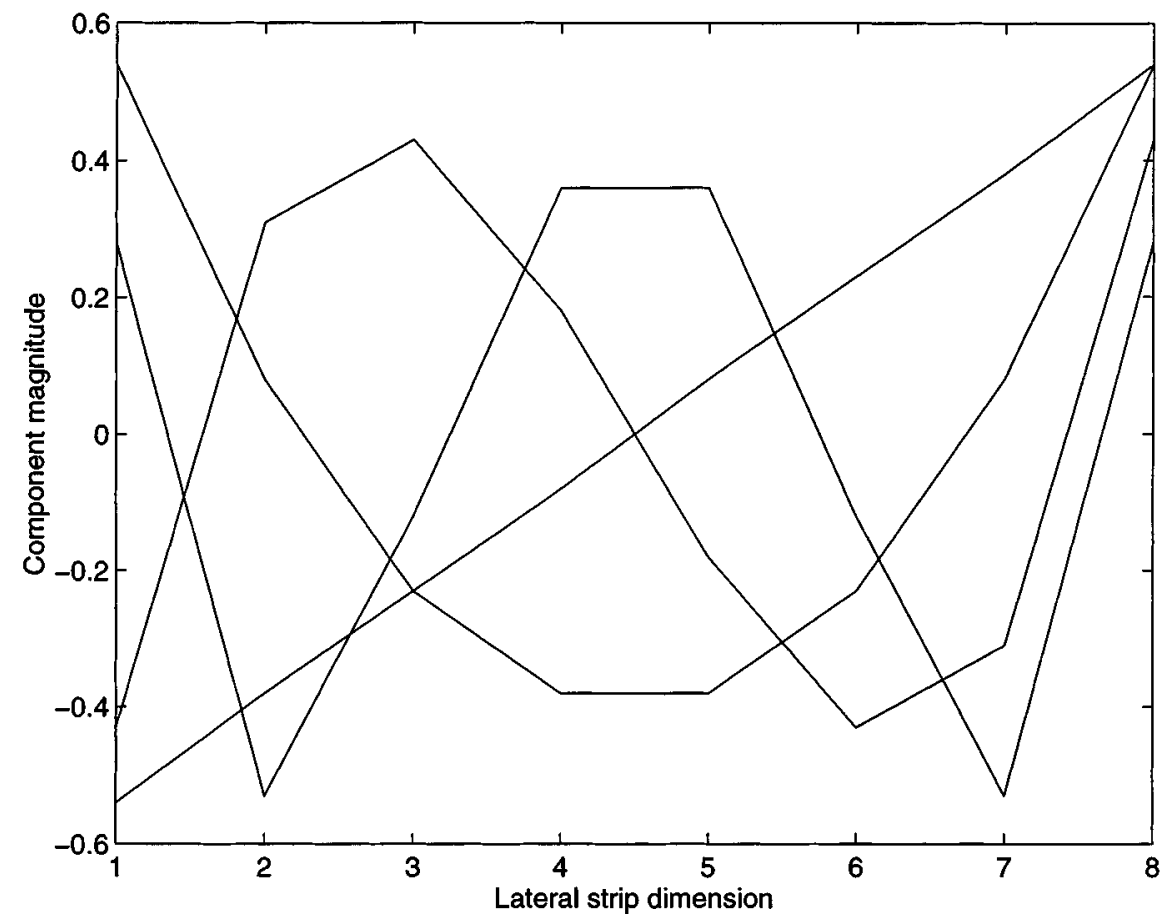

Fig. 3. First- to fourth-order Gram polynomials.

shape profile and limit the roll-bending to fourth order by reducing the number of control inputs to four. From a more theoretical standpoint, the parameterization has effectively reduced the dimension of the transfer function matrix, thus circumventing the singularity problem. Consequently, the mill TFM in (11) may now be diagonalized by application of a "static" precompensator as

$$
K_{0}=\left(P G_{a} P^{T}\right)^{-1} \in \mathbb{R}^{4 \times 4} .
$$

The mill TFM now reduced to four identical single loops with transfer function $g(s)$, which may be shaped using classical frequency response techniques to obtain suitable transient and steady-state performance. A simple gain-scheduling approach for the dynamic compensator is adopted to cover low $(0 \rightarrow 2)$, medium $(2 \rightarrow 5)$, and high $(5 \rightarrow 15)$ speeds as

$$
k(s)=\frac{k_{1}(1+2 s)}{(1+1000 s)(1+0.9 s)}
$$

with $k_{1}$ as given in Table III. The performance of the system may be examined by observing the response of the system to a disturbance in incoming strip shape profile. The target shape profile is flat, i.e., a uniform stress distribution across the strip. Fig. 4 shows the shape control performance for this design-note that the residual shape profile consists only of high (> fourth-order) components. This is confirmed by the parametric shape variations, also shown in Fig. 4, where "Param. $i$ " indicates the variation of $y_{p}(i)$ in (10).

\section{B. AUR Design 2-Eigenvector Approach}

The design presented in this section is motivated by the singularity associated with the AUR mill matrix and, as such, can
TABLE III

SCHEDULED CONTROLLER GAIN FOR DIFFERENT STRIP SPEEDS

\begin{tabular}{|c|c|c|c|}
\hline Strip speed $(\mathrm{m} / \mathrm{s})$ & $0 \rightarrow 2$ & $2 \rightarrow 5$ & $5 \rightarrow 15$ \\
\hline$k_{1}$ & 100 & 200 & 500 \\
\hline
\end{tabular}

be considered to have its roots in the theoretical domain. From observation of the eigenvector spectrum of $G_{a}$, viz.

$$
\begin{aligned}
\lambda_{i}\left(G_{a}\right)=\left[\begin{array}{llllll}
11.63 & 7.84 & 4.32 & 1.31 & 0.26 & 0.04 \\
-0.03 & -0.03
\end{array}\right], & 1 \leq i \leq 8
\end{aligned}
$$

a separation condition

$$
\mu_{1}=\min _{1 \leq i \leq 4}\left|\lambda_{i}\right| \gg \max _{5 \leq i \leq 8}\left|\lambda_{i}\right|=\mu_{2}
$$

is seen to exist, where the $\lambda_{i}, 1 \leq i \leq 4$, are the eigenvalues associated with the high-order bending, and their small relative magnitude indicate the high gains necessary to set up this type of bending in the mill. Such high-order profiles are systematically ignored by application of a "pseudoinverse" of $G_{a}$. Observe that $G_{a}$ can be expanded as

$$
G_{a}=T_{1} \operatorname{diag}\left(\lambda_{i}\right)_{1 \leq i \leq 4} V_{1}+T_{2} \operatorname{diag}\left(\lambda_{i}\right)_{\tilde{5} \leq i \leq 8} V_{2}
$$

where

$$
T=\left[\begin{array}{ll}
T_{1} & T_{2}
\end{array}\right], \quad T^{-1}=\left[\begin{array}{l}
V_{1} \\
V_{2}
\end{array}\right]
$$

where $T_{1}$ is the $8 \times 4$ matrix of eigenvectors of $G_{a}$ corresponding to the eigenvalues $\lambda_{1}, \cdots, \lambda_{4}$ and $V_{1}$ is the $4 \times 8$ matrix of "dual" eigenvectors. Fig. 5 shows the profile of the eigenvectors corresponding to the four largest eigenvalues. 

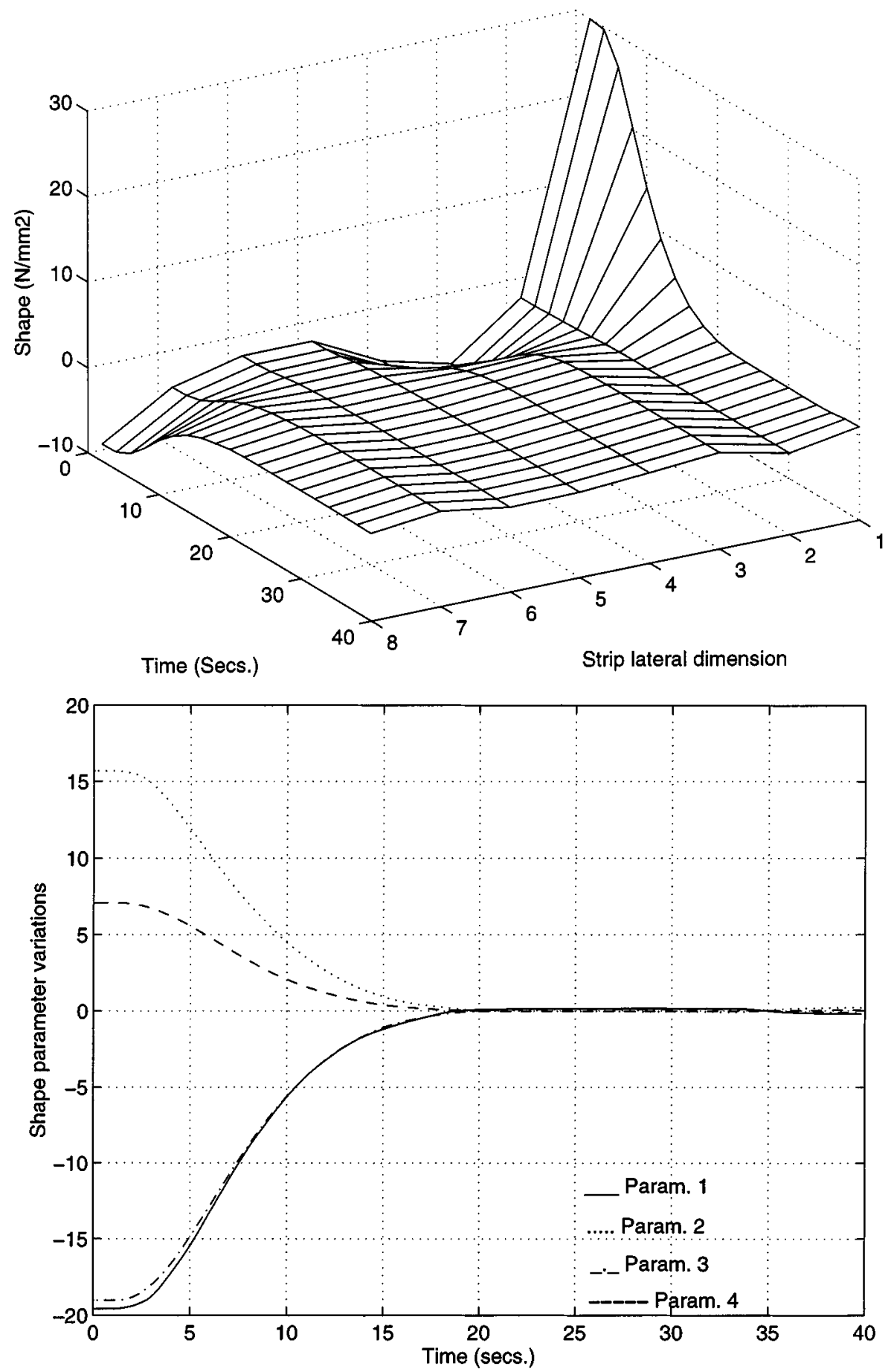

Fig. 4. Shape control and parametric shape variations for AUR Design 1.

Note the similarity (and contrast) with Fig. 3. A controller transfer function matrix is now chosen as

$$
K(s)=T_{1} K_{1}(s) V_{1}, \quad K_{1}(s)=\operatorname{diag}\left[\mathrm{k}_{\mathrm{i}}(\mathrm{s})\right]_{1 \leq \mathrm{i} \leq 4} .
$$

If the same response in each loop is required, the $k_{i}(s)$ can be chosen for simplicity as

$$
\lambda_{i} k_{i}(s)=k(s), \quad 1 \leq i \leq 4
$$

where $k(s)$ is designed to compensate $g(s)$ in (7), as in the design approach presented in Section III-A. Further details on this design methodology are given in [8]. For consistency and to facilitate comparison, $k(s)$ is chosen as in (13). The shape and parametric shape profile variations for this design are showninFig. 6.Itis seen that performance is comparable to that of AUR Design 1.

\section{AUR Design 3-Optimal Control Approach}

An $s$-domain optimal control solution [9] has been proposed for the shape control problem which minimizes the cost functional

$$
\begin{aligned}
J(u)= & \frac{1}{2 \pi j} \int_{-j \infty}^{j \infty}\left(e^{*}(s) L^{*}(s) Q_{1} L(s) e(s)+u^{*}(s)\right. \\
& \left.\cdot R_{1} u(s)\right) d s
\end{aligned}
$$




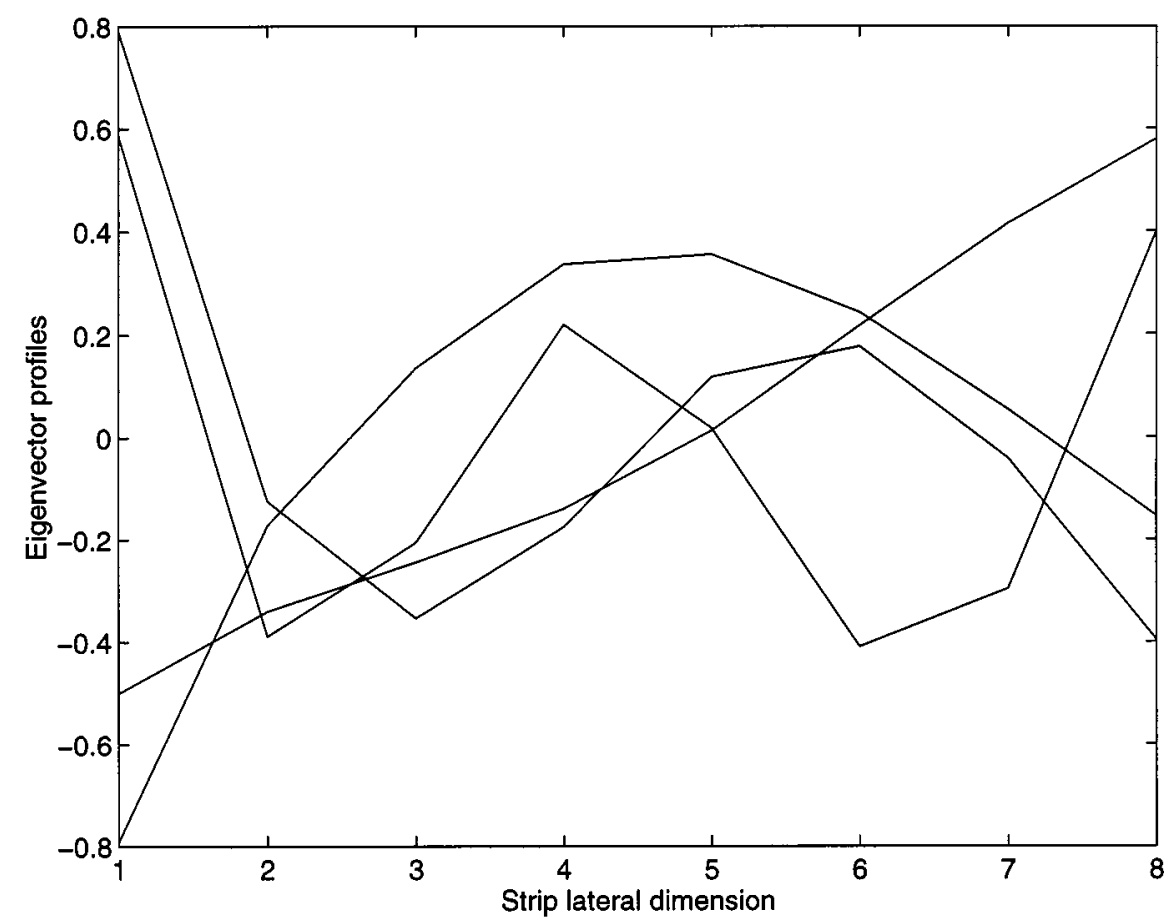

Fig. 5. Eigenvector profiles for $T_{1}$ matrix.

where the superscript ${ }^{*}$ denotes the adjoint operator [e.g., $L^{*}=$ $\left.L^{T}(-s)\right]$, where

$$
\begin{aligned}
& Q_{1}=Q^{T} Q \geq 0 \text { is the error weighting, and } \\
& R_{1}=R^{T} R>0 \text { is the control weighting }
\end{aligned}
$$

$L(s)$ is a linear dynamical operator and allows for the provision of integral action, which may be necessary if the error weighting is made small relative to the control weighting. However, since conservation of control energy is not an issue here (although observation of actuator constraints is), the solution will focus predominantly on performance. Welds in the incoming steel strip can cause step changes in the shape profile, so the system will be optimized for step changes in reference and disturbance inputs via the following choices:

$$
\begin{gathered}
r(s)=\frac{k_{1}}{s}, \quad d(s)=\frac{k_{2}}{s} \\
r(s), d(s) \in \mathbb{R}(s)^{8} \quad k_{1}, k_{2} \in \mathbb{R}^{8} .
\end{gathered}
$$

The solution for the closed-loop optimal controller is given [9] as

$$
\begin{aligned}
K_{o}(s)= & {\left[G_{x}^{-1} Q_{1} G_{x}^{-T} D(0)^{T} D(s)-\gamma(s) I_{x}\right]^{-1} } \\
& \cdot G_{x}^{-1} \sigma(s)
\end{aligned}
$$

where

$$
\begin{aligned}
\frac{D^{*}(s) D(s)}{\sigma^{*}(s) \sigma(s)} & =G^{*}(s) Q_{1} G(s)+R_{1} \\
G(s) & =\frac{\gamma(s)}{\sigma(s)} G_{x} .
\end{aligned}
$$

$G_{x}$ is used to denote the mill matrix (of appropriate dimenstion $x)$. Note that since $K_{o}(s)$ in (22) contains an inverse of the mill matrix, a reduced matrix [as in (11)] must be used. However in spite of this inverse, the optimal controller does not try to diagonalize the system, since the term within the square brackets is nondiagonal. However, if the error weighting matrix $Q_{1}$ is chosen to be of the form

$$
Q_{1}=G_{x}^{-T} Q_{0} G_{x}^{-1}
$$

where $Q_{0}, R_{1}$ are diagonal matrices and the normalized zero frequency gain in each loop is unity, then $K_{o}(s)$ reduces to

$$
K_{o}(s)=\frac{q \sigma(s)}{d(s)(q+r)^{1 / 2}-q \gamma(s)} G_{x}^{-1}
$$

with

$$
d^{*}(s) d(s)=q \gamma^{*}(s) \gamma(s)+r \sigma^{*}(s) \sigma(s)
$$

and

$$
\gamma(0)=\sigma(0)=1, \quad K_{o}(0)=\frac{q}{r} G_{x}^{-1} .
$$

Such a choice for $Q_{1}$ accords with weighting the transformed shape error profile, which are the errors which the actuators must correct and it is important to limit these errors because of the constraints on actuator movement. The solution in (25) may be justified physically, since the plant pole polynomial is cancelled and $G_{x}^{-1}$ in the controller results in four effective single loops. An example calculation with $q=100$ and $r=1$ gives a medium speed scalar controller of

$$
\begin{gathered}
45.86 s^{4}+285.58 s^{3}+550.45 s^{2} \\
k_{o}(s)=\frac{+409.56 s+100.0019}{46.32 s^{4}+288.43 s^{3}+555.95 s^{2}} \\
+442.76 s+1.0019 .
\end{gathered}
$$

Note that a first-order Padé approximation must be used for the delay in the controller calculation. Fig. 7 shows the shape variations for this choice of controller, with a parameterization as in Section III-A. The principal feature is the more aggressive 

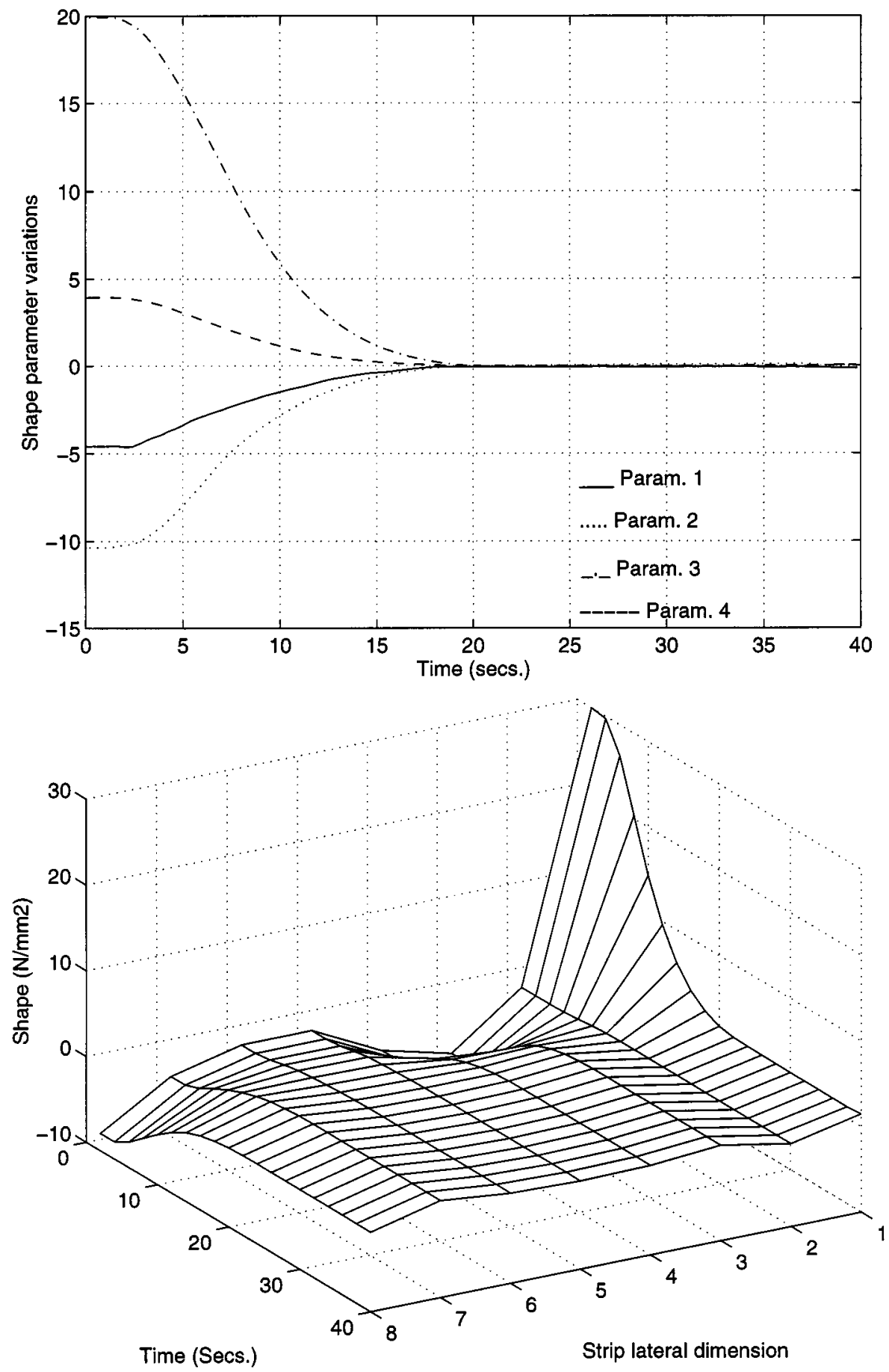

Fig. 6. Shape control and parametric shape variations for AUR Design 2.

transient response, achieved through the large relative value for error weighting.

\section{COMBINED AUR/FIR CONTROL SYSTEM DESIGN}

In this section, the control system will be expanded to include the FIR shape actuators. These devices can have a considerable effect on shape at the strip edges and the proximity of the FIR's to the strip, combined with their relatively large range of travel ( $\pm \pm 110 \mathrm{~mm}$ as opposed to $\pm 50 \mathrm{~mm}$ for the AUR's) give them considerable influence over strip shape. Note that the elements of $G_{i}$ are of the same order of magnitude as $G_{a}$. For combined AUR/FIR operation, it is especially important that an actuator linearization technique be employed (with the same $\tau_{e}$ ), since they have considerably different operating speeds and parameters [2].

\section{A. AUR/FIR Design 1-Multilevel Approach}

The motivation for this approach arises from rolling practice, where (in the main) the AUR's are used to control low-order shape profiles and the FIR's are used to control higher order 

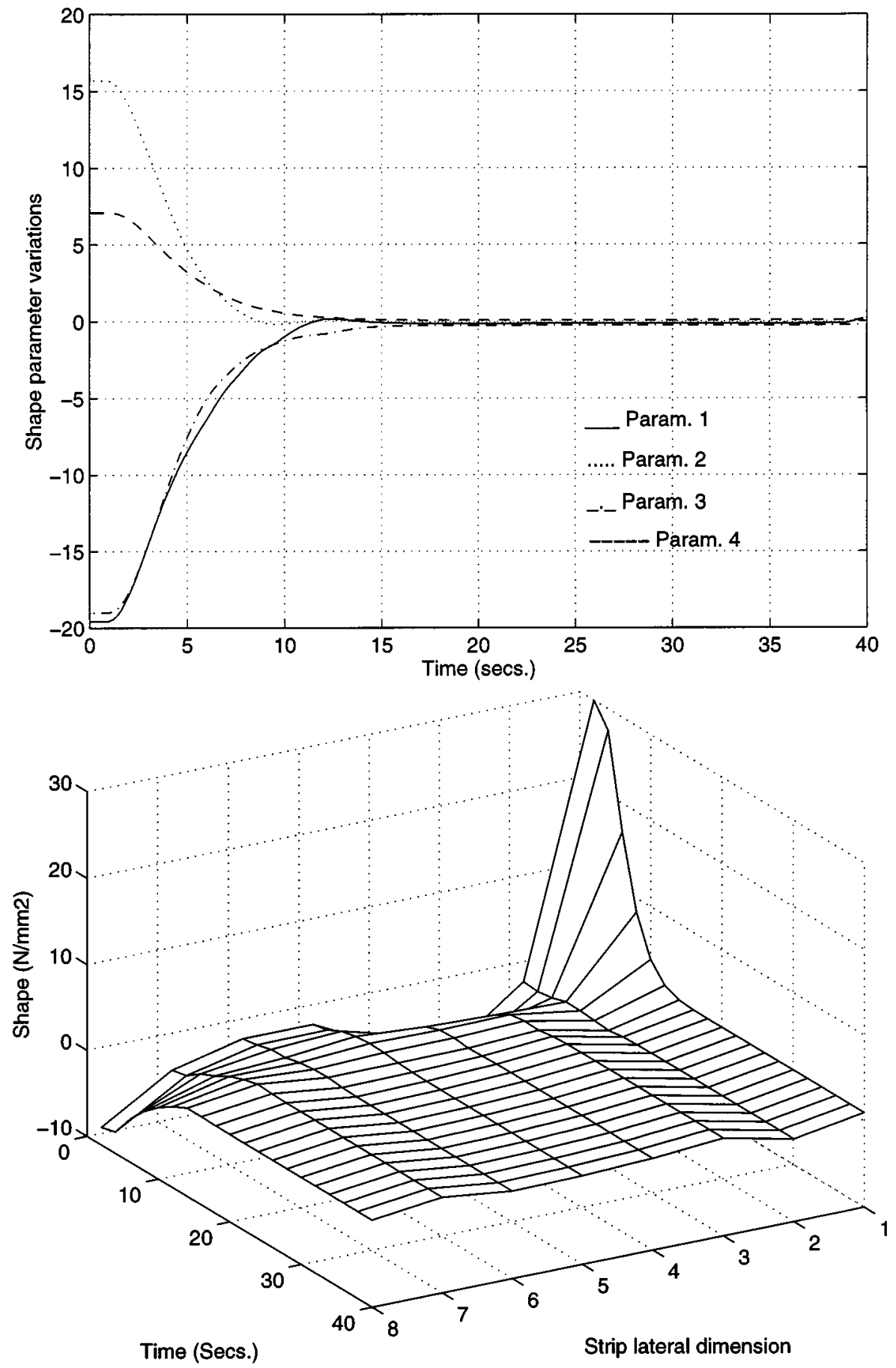

Fig. 7. Shape control and parametric shape variations for AUR Design 3.

profiles. Under such a regime, the restrictions regarding relative AUR movements are not violated and the best potential of the FIR's are realized, since their influence is greatest at the strip edges (high-order variations). However, the methology here is slightly more general in that any two shape parameter coefficients may be controlled by each actuator set, providing FIR and AUR parameterizations are mutually orthogonal. The Gram polynomials, as used in Section III-A, will also be utilized here for shape profile parameterization. The matrix $P_{1}$ will be used to represent the AUR parameterization, and $P_{2}$ the FIR parameterization, where $P_{1}, P_{2} \in \mathbb{R}^{2 \times 8}$. The reasoning behind the multiloop structure is relatively straightforward. Since the FIR system has only two inputs, it can, at most, control only two shape parameter coefficients. The FIR loop, therefore, is chosen as the independent loop, and is diagonalized with respect to the parameter set $P_{2}$ in the arrangement shown in Fig. 8, using $K_{1} \in \mathbb{R}^{2 \times 2}$. However, some undesirable shape components in the range space of $P_{1}$ are produced at the roll-gap by the FIR's, since they have no control over this parameter set. From knowledge of $G_{i}$, these components may be evaluated, and the parameter demand in the AUR loop adjusted accordingly via the cross-coupling term $K_{i a} \in \mathbb{R}^{2 \times 2}$. 


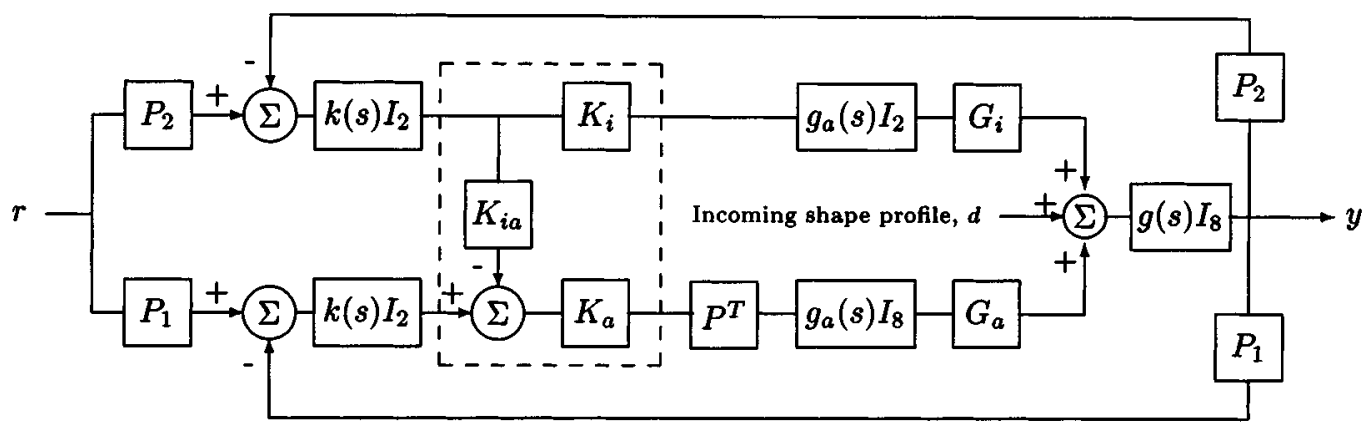

Fig. 8. Multiloop structure.

The AUR loop (dependent loop) can, unlike the FIR's, control all four parameter coefficients, since it has eight inputs (reduced to four by the parameterization). The demand in the parameter coefficients corresponding to $P_{2}$ is set to zero, therefore ensuring that no undesirable shape components in the range space of $P_{2}$ are produced at the roll-gap by the AUR's. The AUR controller, $K_{a}^{*} \in \mathbb{R}^{4 \times 4}$ as a result, has four inputs, two of which are zero. An expression for the equivalent two-input-four-output controller, $K_{a} \in \mathbb{R}^{4 \times 2}$ (shown in Fig. 8), will also be given.

Theorem: The system is diagonalized by the choice of controllers $K_{i}, K_{a}^{*}$, and $K_{i a}$ as follows:

$$
\begin{aligned}
K_{i} & =\left(P_{2} G_{i}\right)^{-1} \in \mathbb{R}^{2 \times 2} \\
K_{a}^{*} & =\left(P G_{a} P^{T}\right)^{-1} \in \mathbb{R}^{4 \times 4}
\end{aligned}
$$

where

$$
P=\left[\begin{array}{ll}
P_{1}^{T} & P_{2}^{T}
\end{array}\right]^{T}
$$

and

$$
K_{i a}=P_{1} G_{i}\left(P_{2} G_{i}\right)^{-1} \in \mathbb{R}^{2 \times 2} .
$$

The proof is given in [10]. This reference also proves the relation

$$
K_{a}=\left[\begin{array}{c}
\left(Q_{1}-Q_{2} Q_{4}^{-1} Q_{3}\right)^{-1} \\
\left(Q_{2}-Q_{1} Q_{3}^{-1} Q_{4}\right)^{-1}
\end{array}\right] \in \mathbb{R}^{4 \times 2}
$$

where

$$
\begin{aligned}
\left(P G_{a} P^{T}\right)^{-1} & =\left(\begin{array}{ll}
P_{1} G_{a} P_{1}^{T} & P_{1} G_{a} P_{2}^{T} \\
P_{2} G_{a} P_{1}^{T} & P_{2} G_{a} P_{2}^{T}
\end{array}\right)^{-1} \\
& =\left(\begin{array}{ll}
Q_{1} & Q_{2} \\
Q_{3} & Q_{4}
\end{array}\right)^{-1} .
\end{aligned}
$$

Note that an overall controller matrix (as indicated by the dashed box in Fig. 8) may be identified as

$$
K_{m l}=\left[\begin{array}{cc}
K_{a} & -K_{a} K_{i a} \\
0 & K_{i}
\end{array}\right] \in \mathbb{R}^{6 \times 4} .
$$

The performance of the multilevel controller is evaluated for the choice of

where

$$
P_{1}^{T}=\left(\begin{array}{ll}
p_{1} & p_{2}
\end{array}\right), \quad P_{2}^{T}=\left(\begin{array}{ll}
p_{3} & p_{4}
\end{array}\right)
$$

$$
P^{T}=\left(\begin{array}{llll}
p_{1} & p_{2} & p_{3} & p_{4}
\end{array}\right), \quad p_{1}, p_{2}, p_{3}, p_{4} \in \mathbb{R}^{8} .
$$

This accords with rolling practice, as discussed earlier. The shape profile variations are shown in Fig. 9 using a $k(s)$ as determined in (28).

\section{B. AUR/FIR Design 2-Unified Approach}

In this approach, the allocation of shape parameters to each actuator set is performed in an automatic and, in some sense, "optimal" manner. Starting with (6), a parameterized mill TFM may be obtained as

$$
G_{p}(s)=g(s) P\left[G_{a} P^{T} G_{i}\right]=g(s) G_{p}(0) \in \mathbb{R}(s)^{4 \times 6} .
$$

Let a right inverse, $K_{r i}$, be defined such that

$$
\left[P G_{a} P^{T} \quad P G_{i}\right] K_{r i}=I_{4}
$$

which reduces the system to four identical single input-single output (SISO) systems in parallel. It can be shown [7] that $P G_{a} P^{T}\left(\in \mathbb{R}^{4 \times 4}\right)$ is full rank and hence that $G_{p}(0)$ is full row rank. Therefore, a right inverse, $K_{r i}$ exists, but is not necessarily unique. One way of exploiting the resulting design freedom is to minimize the norm of the control inputs to the actuators. This helps to ensure that actuator wear is kept to a minimum and that the actuators do not attempt to violate their relative positional restrictions. The required right inverse which minimizes $u^{T} u$ is evaluated as

$$
K_{r i}=G_{p}(0)^{T}\left(G_{p}(0) G_{p}(0)^{T}\right)^{-1} .
$$

A proof, using Lagrange multipliers, is given in [11]. This choice of right inverse produces shape control performance as shown in Fig. 10. Again the $k(s)$ of (28) was utilized. Note that the controller matrix of (34) also constitutes a right inverse of $G_{p}(0)$, but having different properties to that in (39).

\section{AUR/FIR Design 3-SVD Approach}

The approach in this section is motivated by the philosophy in Section III-B which utilized a "natural" parameterization for the system. Since the structure currently being addressed is nonsingular, the singular value decomposition (SVD) is appropriate as a decomposition tool in favor of the eigenvalue decomposition of Section III-B. A benefit of the SVD approach is that an SVD approach provides a natural basis for the treatment of robustness calculations. From the SVD spectrum shown in (8), a separation similar to that in (15) may be identified. In view of this, $G(s)$ [as in (6)] may be decomposed as

$$
G(s)=g(s)\left[U_{1} \Sigma_{1} V_{1}^{T}+U_{2} \Sigma_{2} V_{2}^{T}\right]
$$

where $\Sigma_{1}$ contains the four "large" singular values and $\Sigma_{2}$ the four "small" ones. The profile of $U_{1}$ (containing the singular vectors corresponding to $\Sigma_{1}$ ), which parameterizes the shape profile, is similar to that shown in Figs. 3 and 5. A controller 

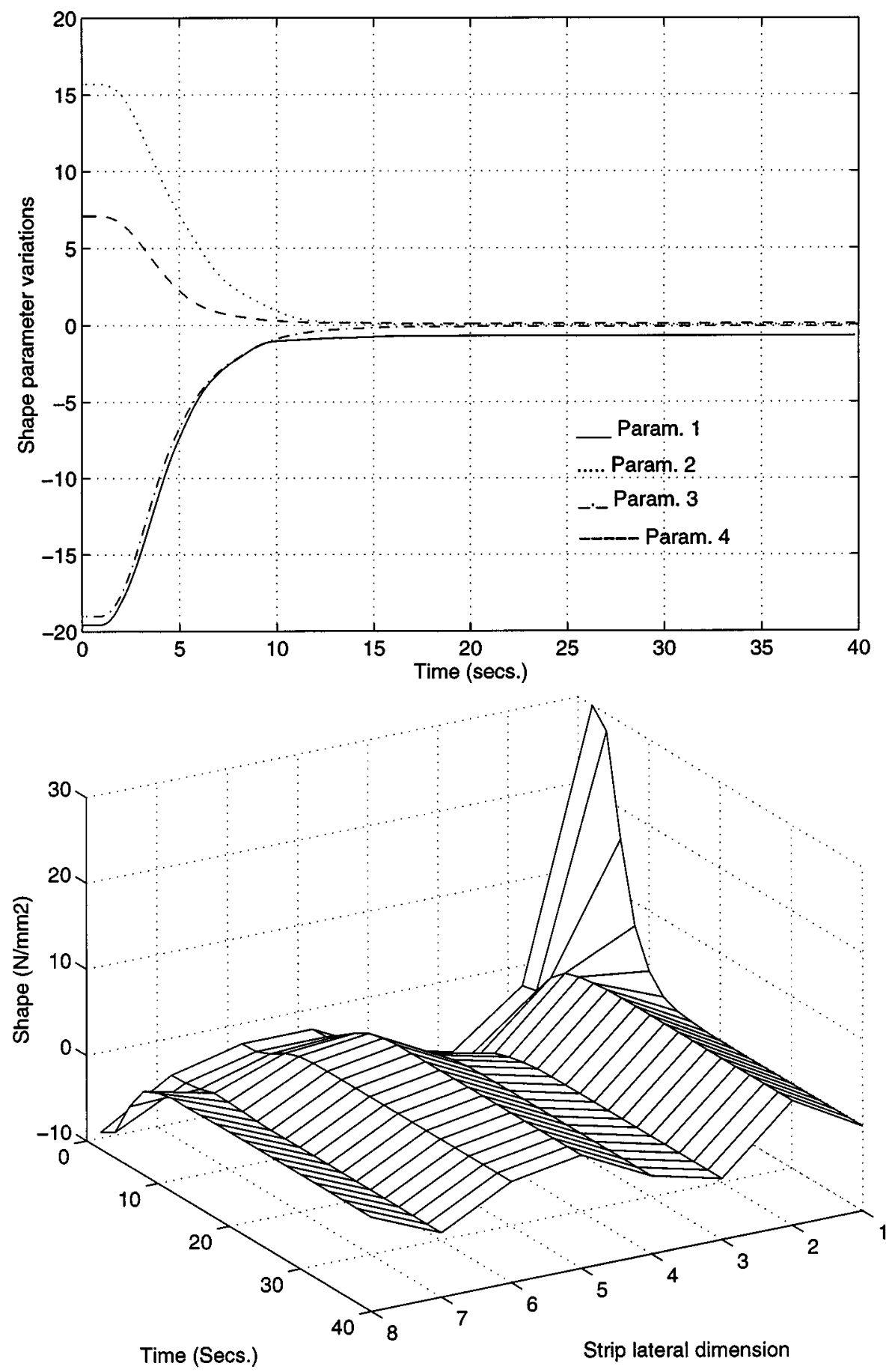

Fig. 9. Shape control and parametric shape variations for multiloop controller.

which deliberately ignores the shape errors corresponding to the $\Sigma_{2}$ singular values may now be stated as

$$
K(s)=k(s) K=k(s) V_{1} \Sigma_{1}^{-1} U_{1}^{T} .
$$

Using the $k(s)$ in (28), the parametric shape profiles obtained using the SVD controller are shown in Fig. 11. Further details of this design are given in [12].

\section{FEEDFORWARD CONTROL}

It has been shown [13]-[15] that the input shape profile is strongly reproduced in the output strip. Due to the reversing nature of the $\mathrm{Z}$ mill, shapemeters exist at both sides of the roll-gap, facilitating measurement of the incoming strip shape (for feedforward control) while the output shapemeter is being used for feedback control. Currently, however, only one set of shapemeter electronics is available, which is switched between the two shapemeters, depending on the direction of strip travel. Although feedforward control can provide some anticipative action for poor incoming strip shape, it cannot, on its own, guarantee the quality of output strip shape, since:

- nonuniform rolls, thermal and ground camber impart a residual shape profile to the strip; 

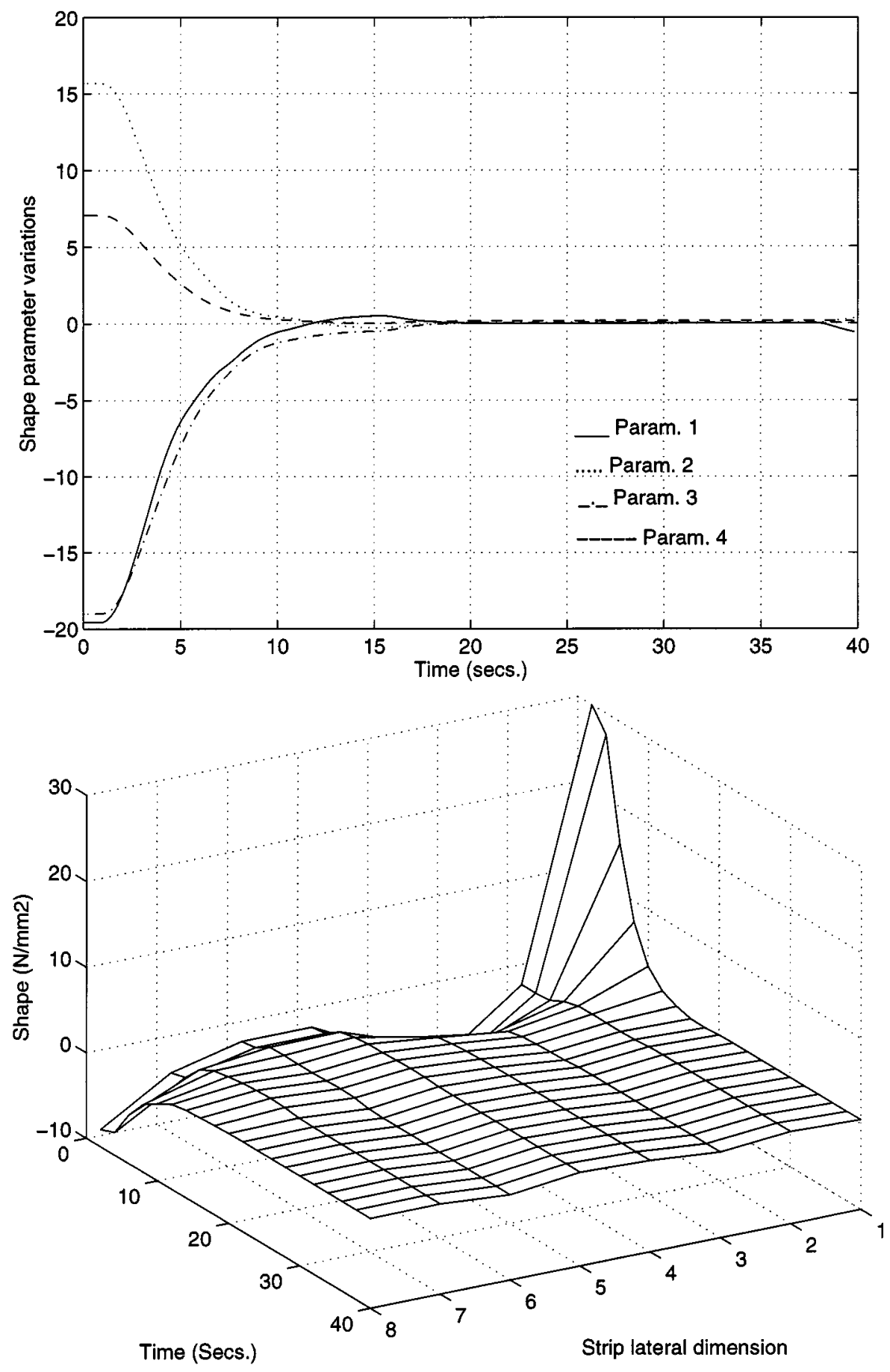

Fig. 10. Shape control and parametric shape variations for unified controller.

- modeling inaccuracies in $G_{m}$ mean that an exact inverse shape profile (to the incoming strip) cannot be realized;

- mismatch between the incoming gauge profile and the roll-gap profile will cause shape defects;

- the nonlinear nature of the actuators will introduce timing problems in the application of an inverse profile.

Nevertheless, there are advantages which may be gained from measurement of strip shape $2.91 \mathrm{~m}$ before it enters the roll-gap, rather than $2.91 \mathrm{~m}$ after the roll-gap, since effectively 2.91 $\mathrm{m}$ (at least) of the strip remains uncontrolled by feedback control.
For illustration, a feedforward scheme utilizing the AUR's only (for the sake of brevity) will be considered. However, since a nonsquare inverse of $G_{m}$ has been dealt with in Section IV, there is no difficulty in extending the scheme to include the FIR's. Let the shape of the incoming strip be represented by $d \in \mathbb{R}^{8}$. For cancellation of this disturbance, it is required that

$$
d=-G_{a} g_{a}(s) g_{s}(s) K_{f f}(s) e^{\tau_{1} s} d
$$

where the term on the right-hand side must be provided by the controller, while $g_{a}(s)$ and $g_{s}(s)$ represent the (linearized) actuator and shapemeter transfer functions, respectively. The term $e^{\tau_{1} s}$ demonstrates the measurement of the disturbance $d$ 

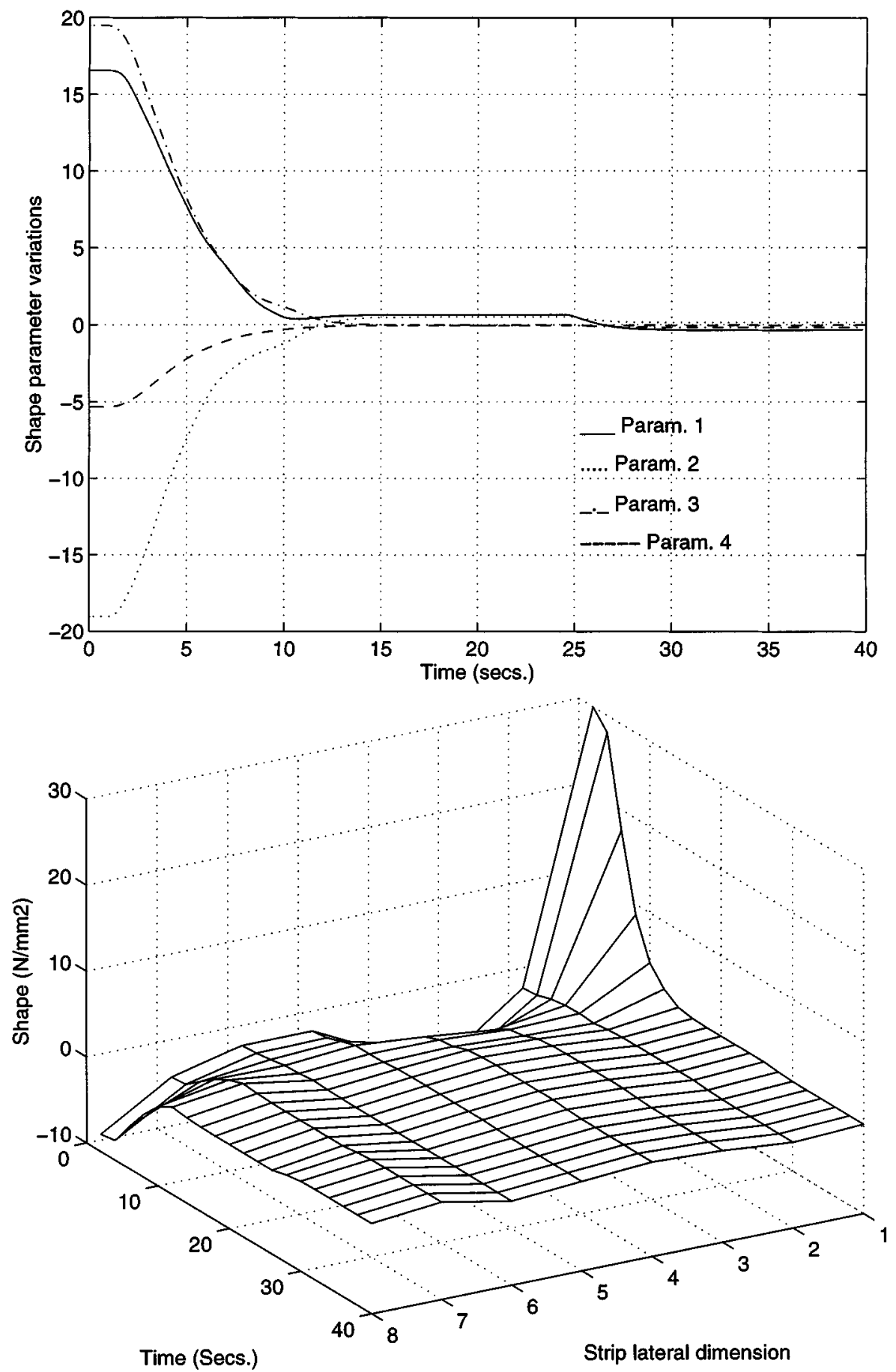

Fig. 11. Shape control and parametric shape variations for SVD controller.

made by the "upstream" shapemeter in advance of it appearing at the roll-gap. From (42), the required feedforward controller. $K_{f f}(s)$, may be determined as

$$
K_{f f}(s)=-\left[g_{a}(s) g_{s}(s) e^{\tau_{1} s}\right]^{-1} G_{a}^{-1}
$$

Since (43) contains an inverse of $G_{a}$, some shape profile parameterization must be employed in order to improve the condition of the mill matrix. As an example, the Gram polynomials [as in (9)] will be employed to obtain a pseudoinverse of $G_{a}$ as

$$
K_{f_{o}}=G_{a}^{-1} \approx P^{T}\left(P G_{a} P^{T}\right)^{-1} P .
$$

where $K_{f o}$ forms the multivariable (but nondynamic) part of $K_{f f}(s)$ as

$$
K_{f f}(s)=K_{f o} k_{f}(s)
$$

where

$$
k_{f}(s)=-\left[g_{a}(s) g_{s}(s) e^{\tau_{1} s}\right]^{-1} .
$$

Since the actuators are rate limited, they cannot be equalized by $g_{a}(s)^{-1}$, but some of the time advance available can be used to 

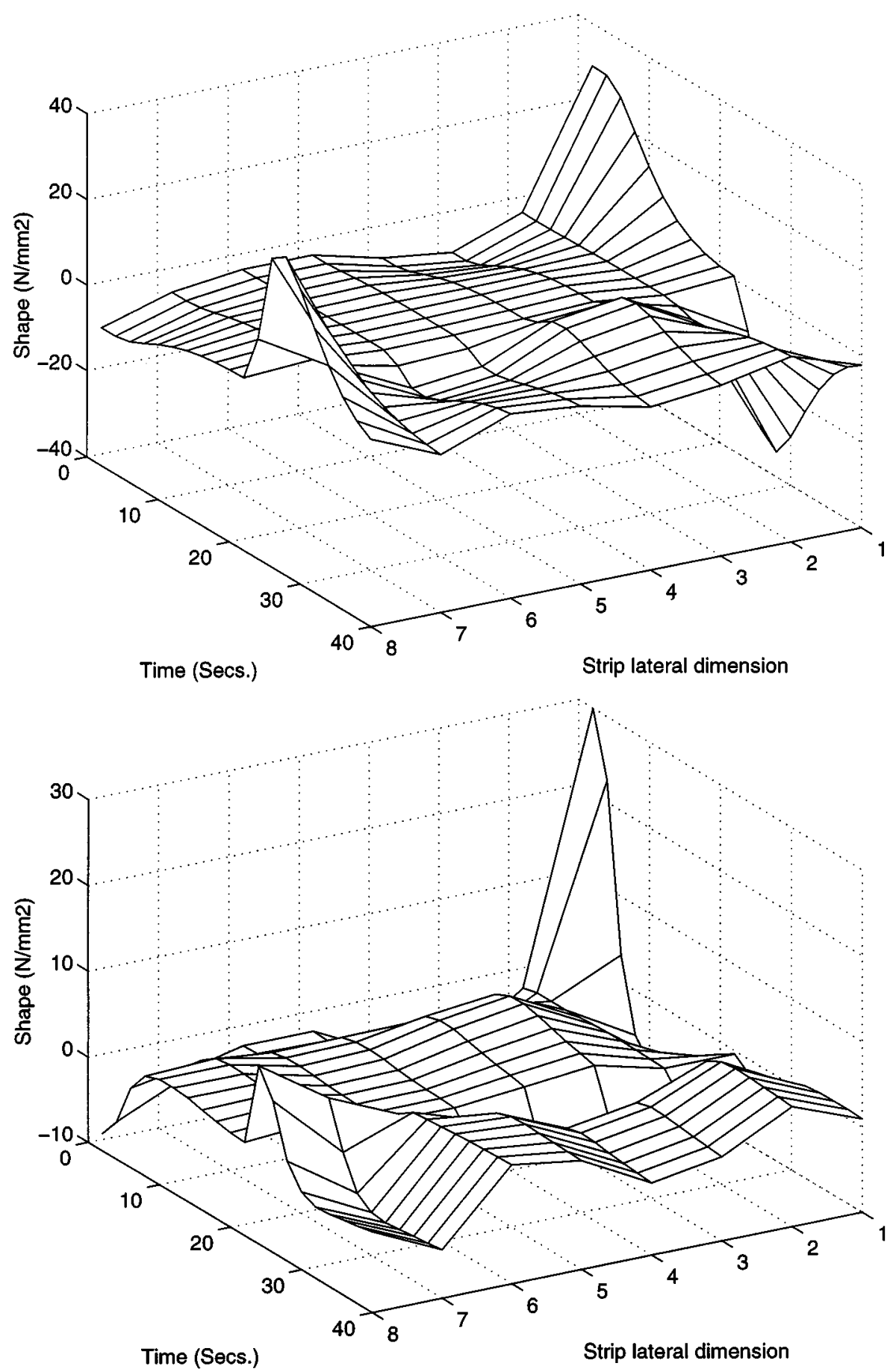

Fig. 12. Shape control performance with/without feedforward.

offset the phse effect of $g_{a}(s)$ in an attempt to get $k_{f}(s)$ close to an all-pass zero-phase element. Least squares [11] was used to determine the optimal advance to offset the phase of $g_{a}(s)$ over the renge $\omega=0 \rightarrow 2 \mathrm{rads} / \mathrm{s}$, while the shapemeter pole is cancelled, with a pole introduced at $s=-100$ for realizability, giving

$$
k_{f}(s)=\frac{(1+0.74 s) e^{-0.14 s}}{(1+0.01 s)} .
$$

To assess the benefits of feedforward, a step change in incoming shape profile was generated at $t=20 \mathrm{~s}$, simulating a weld in the steel strip. A $k(s)$ as in (13) was utilized and Fig. 12 shows the performance for feedback only in part (a), using the controller developed in Section III-A and combined feedback/feedforward in part (b). Table IV shows the comparative performance figures.

\section{System Robustness CALCUlations}

Due to the large number of possible mill matrices, it is important to examine the robustness of the system in the face of perturbations in $G_{a}$ and $G_{i}$. This section includes both passive (cal- 
TABLE IV

Comparative Performance Figures with/without FEEdForWARD

\begin{tabular}{|c|c|c|}
\hline Controller & MSE (parametric) $^{1}$ & MSE (shape) $^{1}$ \\
\hline Feedback only & 142 & 73 \\
\hline Feedback + feedforward & 22 & 14 \\
\hline
\end{tabular}

culations are performed after the controller has been designed) and active (robustness is actively designed into the controller) approaches to dealing with the robustness of the shape control systems. A further solution to the variations in the mill matrix adopts a self-tuning strategy.

\section{A. Robustness Calculations for Existing Controllers}

For a number of the controllers presented in Sections III and IV, robustness calculations have been developed, which describe the allowable perturbations under which system stability is retained. For the AUR designs, calculations for the designs in Sections III-A and III-B are provided in [7] and [8], respectively. For the combined AUR/FIR designs in Sections IV-A-IV-C, robustness calculations are given in [10] and [12], respectively. Since a similar parameterization to that in Section III-A is utilized in Section III-C, AUR Design 3 is covered by the calculations in [7]. To give an impression of the nature of these calculations, robustness measures for AUR Design 2 (decomposition-based) and AUR/FIR Designs 2 and 3 (parameterization) are given here, which convey the spirit of the approach.

AUR Design 2: Stability is retained, provided

$$
1>\sum_{p=1}^{8} \sum_{q=1}^{8} c_{r p q}\left|\Delta_{p q}\right|, \quad 1 \leq r \leq 4
$$

where $\Delta_{p q}$ are the elements of the perturbation matrix $\Delta$ in $G_{a}$ and $c_{r p q}$ are scalars described by

$$
c_{r p q}=\sup _{\omega \geq 0}\left|\frac{g(s) k(s)}{1+g(s) k(s)}\right| d_{r p q}
$$

where

$$
d_{r p q}=\sum_{j=1}^{4}\left|\lambda_{1}^{-1}\right|\left|\left(V_{1}\right)_{r p}\left(T_{1}\right)_{q j}\right|
$$

Some interesting observations regarding this result can be made.

- The $c_{r p q}$ are proportional to $\lambda_{r}^{-1}$, indicating that small eigenvalues reduce the permissable perturbation $\Delta$.

- The properties of the scalar c.l.t.f., $g k(s) /(1+g k(s))$ clearly affect stability. If, for example, it posesses a strong resonance, $c_{r p q}$ will be large, increasing sensitivity to the perturbation $\Delta$. This has implications for more highly tuned controllers, such as the optimal controller of Section III-C.

AUR/FIR Designs 1 and 2: The stability condition here is also expressed as a set of linear inequalities as

$$
1>\sum_{j=1}^{4}\left|\gamma(\Delta K)_{r j}\right|, \quad 1 \leq r \leq 4
$$

where

$$
\gamma=\sup _{\omega \geq 0}\left|(1+g k)^{-1} g k\right|
$$

$\Delta$ is the perturbation in the parameterized mill matrix, $G_{p}(0)$ [as in (37)] and $K=K_{m l}$ for the multilevel controller [as in (34)], with $K=K_{r i}$ for the right inverse controller [as in (39)].

Equations (48) and (51) allow calculation of the range of mill matrices under which stability is retained by a nominal controller. They give no indication, however, of what deterioration in performance may take place.

\section{B. Development of a Robust Controller}

This section directly addresses the issue of utilizing a single controller to cover all the passes of a schedule (or possible a number of schedules) and making the controller optimally insensitive (in a $H_{\infty}$ sense) to the resulting variations in the mill matrix. For brevity, the concept will use the AUR system only, but is easily extendable to include the FIR system, since a singular value formulation is used. In the $H_{\infty}$ framework, the following cost function is minimized:

where

$$
J=\left|\begin{array}{l}
W_{1} S(s) \\
W_{2} T(s)
\end{array}\right|_{\infty}
$$

$$
S(s)=(1+G K(s))^{-1}
$$

is the system sensitivity function, which determines the disturbance rejection properties of the system, and

$$
T(s)=G K(s)(1+G K(s))^{-1}
$$

is the complementary sensitivity function, which determines robust stability and measurement (shapemeter) noise attenuation. Tradeoffs and conflicts arising from these different requirements are resolved using the weighting functions $W_{1}(s)$ and $W_{2}(s)$. A further issue in weight selection is that the closed-loop bandwidth rolls off in frequency before the phase effect of the pure delay term in $g(s)$ become significant. Robust stability is guaranteed by ensuring that the weight $W_{2}(s)$ overbounds the plant (multiplicative) perturbation in the max. singular value sense as

$$
\bar{\sigma}\left[W_{2}(j \omega)\right] \geq \bar{\sigma}[\Delta(j \omega)]
$$

where

$$
G(s)=G_{n o m}(s)(I+\Delta(s)) .
$$

Inclusion of the full $8 \times 8$ system poses a significant problem for $H_{\infty}$ design, since the sensitivity function $S(s)$, will always be close to unity in the directions of the small singular values [i.e., the product $G H(s)$ is approximately zero in these directions]. The plant is therefore parameterized (as before) in terms of the four most significant singular values from the spectrum

$$
\begin{array}{r}
\sigma_{i}\left(G_{a}\right)=\left[\begin{array}{llllll}
12.35 & 9.11 & 4.91 & 1.56 & 0.33 & 0.21 \\
0.025 & 0.0051
\end{array}\right], \\
1 \leq i \leq 8
\end{array}
$$

as in (40), but in this case with

$$
U_{1}, U_{2}, V_{1}, V_{2} \in \mathbb{R}^{8 \times 4}, \quad \Sigma_{1}, \Sigma_{2} \in \mathbb{R}^{4 \times 4} .
$$

The weighting functions are now chosen as

$$
\begin{aligned}
& W_{1}(s)=\frac{10\left(10^{-5} s+1\right)}{100 s+1} I_{4} \\
& W_{2}(s)=\frac{0.2774\left(10^{-3} s+1\right)}{\left(10^{-6} s+1\right)} .
\end{aligned}
$$

$W_{1}(s)$ is chosen to: 


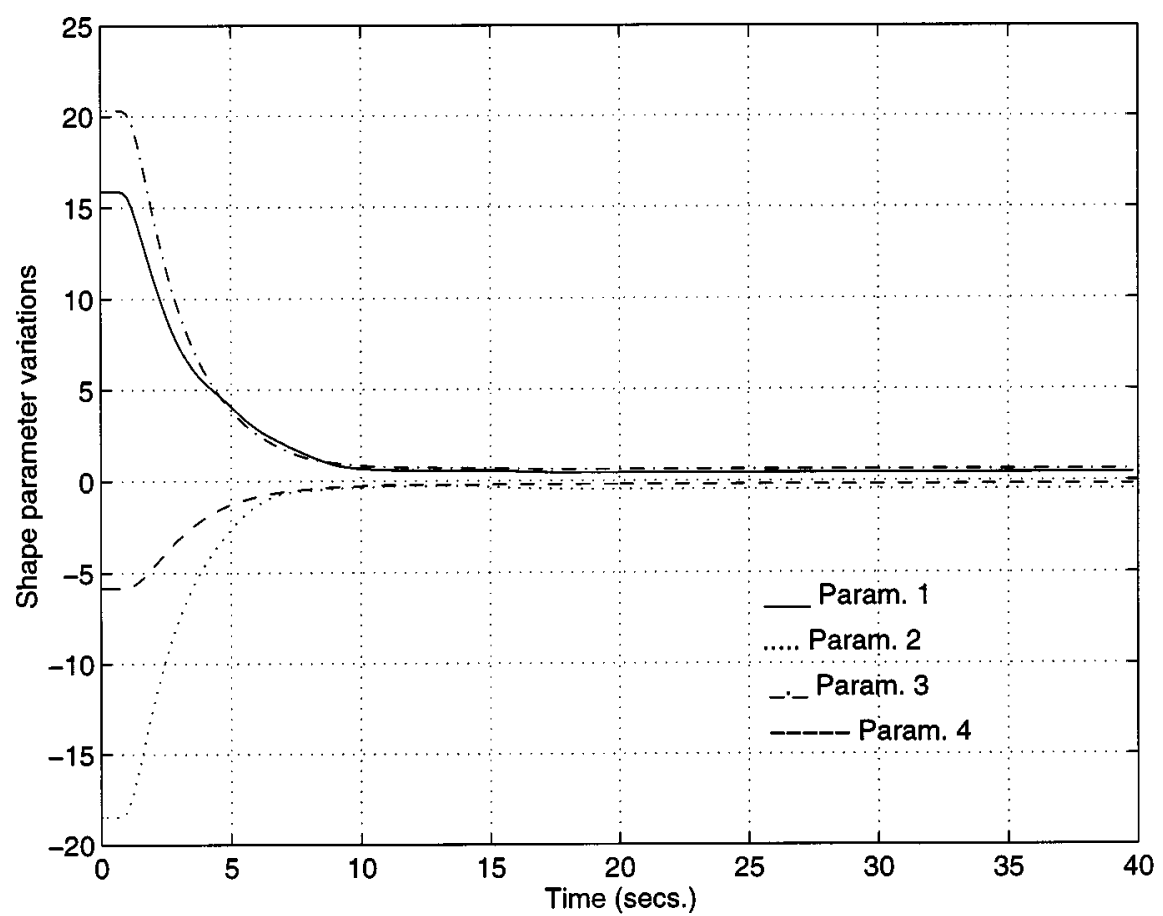

(a)

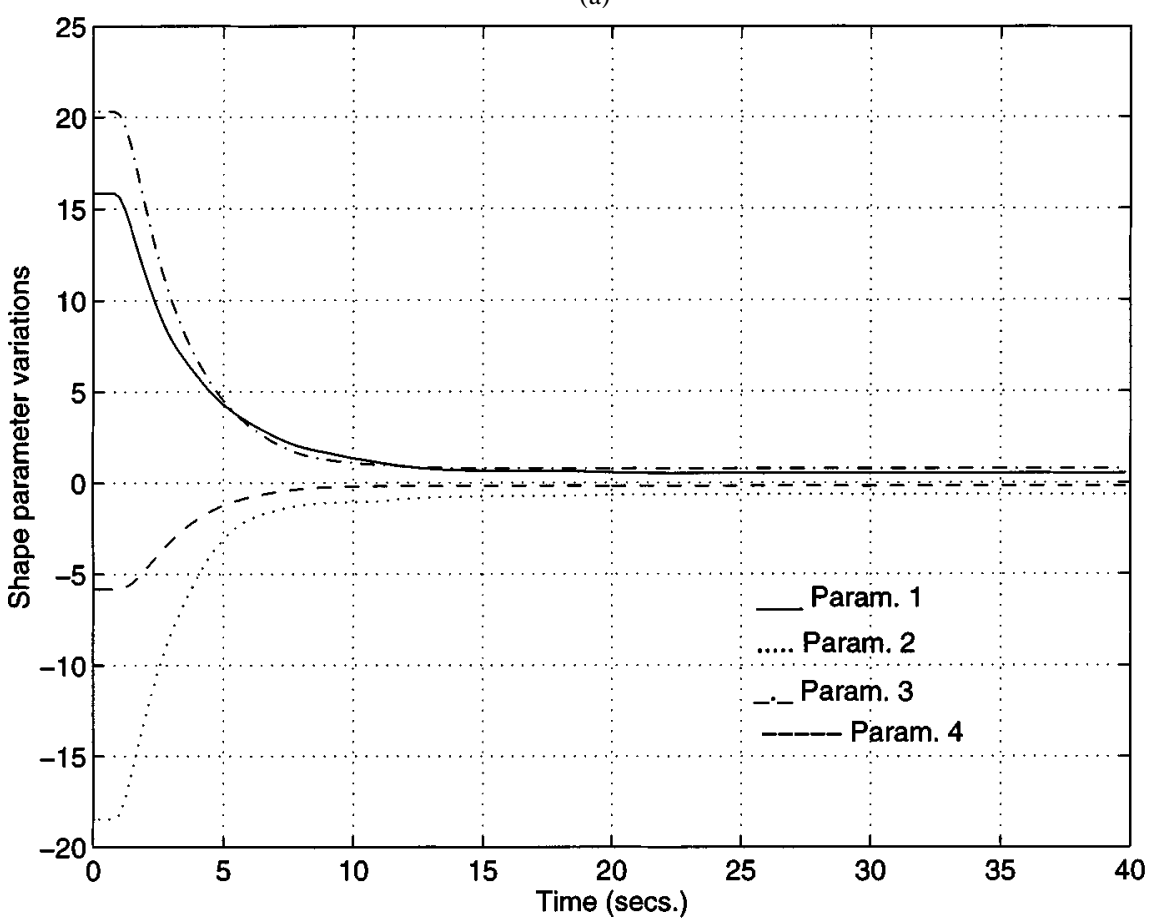

(b)

Fig. 13. Parametric shape variations for nominal and perturbed $G_{a}$.

- penalize sensitivity, $S(s)$, at low frequency, giving good d.c. disturbance rejection;

- ensure that the dynamic response is maintained in spite of parameter variations at low frequency.

$W_{2}(s)$ is chosen to:

- ensure robust stability by covering $\Delta(s)$, i.e., that condition (56) is met;

- attenuate high frequency (shapemeter) measurement noise, by driving $T(s)$ down at high frequency.
Further details on the $H_{\infty}$ design are provided in [16]. The comparative performances of the $H_{\infty}$ controller on the nominal system (an averaged $G_{a}$ over the six passes of a schedule) and that for Pass 6 are shown in Fig. 13 in (a) and (b), respectively. A quantitative comparison is given in Table $\mathrm{V}$.

\section{Self-Tuning/Adaptive Control}

A further solution to variations in the mill matrix is to adopt a self-tuning/adaptive strategy [17]. Such a methodology uses 
TABLE V

PERformance Figures For $H_{\infty}$ CONTROLleR

\begin{tabular}{|c|c|c|}
\hline$G_{a}$ & MSE (parametric) & MSE (shape) \\
\hline Nominal (av.) & 15.18 & 8.84 \\
\hline Pass 6 & 16.06 & 8.85 \\
\hline
\end{tabular}

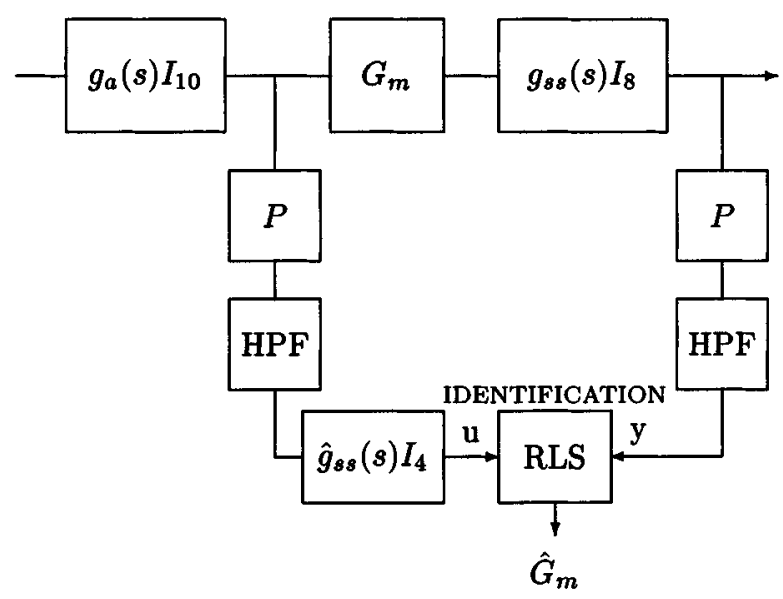

Fig. 14. On-line dentification of $G_{m}$.

measurements of the actual actuator positions and the measured shape profile to construct an on-line model of the plant. Furthermore, since the dynamics of the system are well understood, the identification is focussed on measurement of the mill matrix, in the configuration shown in Fig. 14. Since all of the control schemes presented utilize some form of parameterization, it is appropriate to identify only a reduced mill matrix. One further addition is the use of high-pass filtering on output and input signals to remove the effect of the disturbance from the output signal. Both AUR and combined AUR/FIR systems can be dealt with and explicit or implicit controller forms can be constructed. In the explicit version, a reduced mill matrix is identified and the controller calculated on-line from (for example) (39) or (12). In the implicit scheme, an inverse of the reduced mill matrix may be obtained directly by interchanging the inputs $(y \leftrightarrow u)$ to the identification algorithm. One interesting feature of the combined AUR/FIR implicit scheme, which employs a nonsquare matrix inverse, is that (after initial parameter convergence) the recursive parameter estimates do not converge to constant values, but at each point in time provide a right inverse, exploiting the nonuniqueness of the solution. If a unique solution is required, for example one which minimizes $u^{T} u$, an explicit scheme can be employed utilizing (for example) (39). Both explicit and implicit results for AUR and combined AUR/FIR systems are given in [3] and [17].

\section{CONCLUSIONS}

This paper has attempted to demonstrate some of the components which may be used to assemble a shape control system for a Sendzimir mill. The most important issues to be dealt with in the formulation of control strategy are the following.

1) Actuatorlinearization. Thisisimportant, sinceall the controller formulations employed in this paper require a linear model. In addition, since AUR and FIR actuators operate at
TABLE VI

RELATIVE CONTROL EFFORT FOR DIFFERENT AUR/FIR STRATEGIES

\begin{tabular}{|c|c|c|c|}
\hline Strategy & MSU (AUR) & MSU (FIR) & Parameterisation \\
\hline AUR/FIR Design 1 & 1.6 & 102.6 & Gram \\
\hline AUR/FIR Design 2 & 23.8 & 11.5 & Gram \\
\hline AUR/FIR Design 3 & 19.5 & 7.6 & SVD \\
\hline
\end{tabular}

TABLE VII

Shape Control Performance For DifFERENT Strategies

\begin{tabular}{|c|c|c|c|}
\hline Strategy & MSE (shape) & MSE (parametric) & Parameterisation \\
\hline AUR Design 1 & 20.44 & 39.47 & Gram \\
\hline AUR Design 2 & 20.91 & 20.56 & Eigenvector \\
\hline AUR Design 3 & 12.02 & 22.75 & Gram \\
\hline AUR/FIR Design 1 & 135.39 & 92.47 & Gram \\
\hline AUR/FIR Design 2 & 96.15 & 91.01 & Gram \\
\hline AUR/FIR Design 3 & 99.08 & 95.19 & SVD \\
\hline
\end{tabular}

considerably different speeds $(8 \mathrm{~mm} / \mathrm{s}$ and $3 \mathrm{~mm} / \mathrm{s}$, respectively) they must be equalized before a combined AUR/FIR control system can be designed.

2) Shape profile parameterization. Parameterization of the shape profile reduces the number of shape measurements, concentrates the effort on strip shape characteristics dealt with in normal rolling practice and addresses the singularity problem associated with the mill matrices. A number of parameterizations are possible, including Gram polynomials (Sections III-A, III-C, IV-A, and IV-B), eigensystem decomposition (Section III-B) and singular value decomposition (Sections IV-C and VI-B). In addition, freedom exists in the distribution of shape coefficients to be controlled by different actuator sets in the combined AUR/FIR schemes. Some insight into the choice of parameterization may be had by examining the control effort required for different parameterizations. Table VI compares the mean square control (MSU) for the three AUR/FIR schemes, which all utilize the same dynamic compensator [ $k(s)$ as in (28)]. It would appear that the forced parameter assignment of the multilevel scheme requires extra control effort compared to the right-inverse scheme, but adoption of the parameterization provided by the SVD (which represents the "natural" bending modes presentin the mill)is optimalinterms of ease of control. This has implications for the maximum disturbance amplitudes which can be corrected by the control system. One caveat, however, is that small control signals cause the actuators to operate near their dead-zone region, causing nonlinear behavior, as evidenced by Fig. 11.

3) Robust performance. Since it is impractical to store approximately 3000 different controllers to cover all passes and schedules, it is important that the shape controller be robust to changes in $G_{m}$. In Section VI-B, it was shown that shape parameterization can considerably improve the system sensitivity properties, so each of the control designs presented contain some inherent robustness. However, the $H_{\infty}$ controller goes a step further in shaping the dynamical controller so that "optimal" robustness is 
achieved while attempting to maintain nominal system performance. For controller scheduling, guarantees can be made about controller performance over a given set of plant perturbations (corresponding to different mill matrices), thereby providing a systematic method for developing a range of controllers to cover all schedules. In contrast, the calculations of Section VI-A must be performed exhaustively to check for allowable ranges of perturbations, with no guarantees of performance. The self-tuning/adaptive strategy has the potential to give nominal performance over all passes and schedules, without the need for storage of multiple controllers. The problem generated by the initial convergence period may be somewhat circumvented by starting from the previous mill matrix (explicit) or controller matrix (implicit) values.

Some comments regarding the relative performance of the various controllers are also appropriate. Table VII shows MSEcalclations for all the feedback controllers of Sections III and IV. Note that the systematic (optimal) design procedures (LQ and $H_{\infty}$ ) yield controllers which have superior transient performance. These could be speeded up further (if required) by appropriate choice of weighting functions, subject to the rate limits on the actuators. In particular, the $H_{\infty}$ controller has the best overall MSE figures (see Section VI-B) as well as having its inherent good robustness properties. A consequence of this is that the controller is of high order (order 20), but order reduction techniques could be employed if required, while retaining the essential controller characteristics. From Table VII, and recalling that all AUR/FIR controllers utilize the optimal $k(s)$, there does not seem to be any improvement in system performance by utilizing the FIR's. The main reason for any deterioration in performance is that the same control effort is spread between more actuators, with the actuators having to make smaller movements with correspondingly larger percentage errors in steady-state accuracy (due to dead-zone). However, the additional two actuators available will mean that larger shape disturbances can be handled by combined AUR/FIR schemes.

A final comment relates to controller complexity. The selftuning controller presents the greatest computational challenge, but multiple forms are not required. It is likely that the number of $H_{\infty}$ controllers required to cover the complete operational domain of the mill will be relatively small, compared to the "nonrobust" forms, but at a higher computational cost, since the controller is high order. There would therefore seem to be a tradeoff between computational load and memory requirements, but with current performance/price ratios, implementation of any of the proposed strategies is not seen to be prohibitive.

In conclusion, the SVD approach offers the best parameterization for dealing with the singularity issue, both in terms of the shape control performance and robustness considerations. Variations in mill/strip conditions due to pass or schedule changes can be addressed by either the $H_{\infty}$ controller or an adaptive scheme. Either of these controllers can be implemented using current controller technology, with the adaptive controller requiring complex iterative calculations (in a sampling period of about $40 \mathrm{mS}$ ), while the emphasis is on storage requirements (rather than processing speed) for the robust controller. More pertinent is the issue of controller integrity, where convergence of plant and/or controller parameters may be problematic and potential strip wastage during initial controller parameter convergence. Such considerations would favor the $H_{\infty}$ controller for this important industrial application.

\section{ACKNOWLEDGMENT}

The author would like to all those who have influenced this research work, including Prof. M. J. Grimble and Prof. M. A. Johnson of the University of Strathclyde, Dr. K. Dutton of Sheffield Hallam University (formerly of British Steel), Dr. A. M. Holohan of Dublin City University, and Dr. D. Bates of the University of Leicester (formerly of DCU).

\section{REFERENCES}

[1] G. F. Bryant, Automation of Tandem Mills, J. Iron Steel Inst., 1973.

[2] J. V. Ringwood, "Diagonalization of a class of multivariable system via an actuator linearization technique," in Proc. IMC-11, Belfast, 1994.

[3] — , "The design of shape control systems for a Sendzimir mill," Ph.D. dissertation, University of Strathclyde, 1984.

[4] K. Dutton, "An investigation into the design and performance of a shape control system for a Sendzimir mill," Ph.D. dissertation, Sheffield City Polytechnic, 1983.

[5] J. V. Beck and K. J. Arnold, Parameter Estimation in Engineering and Science. New York: Wiley.

[6] R. A. Fisher and F. Yeates, Statistical Tables for Biological, Agricultural and Medical Research. Edinburgh, U.K.: Oliver and Boyd, 1925.

[7] M. J. Grimble and J. Fotakis, "The design of strip shape control systems for Sendzimir mills," IEEE Trans. Automat. Contr., vol. AC-27, June 1982.

[8] J. V. Ringwood, D. H. Owens, and M. J. Grimble, "Feedback design of a canonical multivariable system with application to shape control in Sendzimir mills," Trans. ASME, J. Dyn. Syst., Meas. and Control, vol. 116, Mar. 1994.

[9] J. V. Ringwood and M. J. Grimble, "An optimal output feedback solution to the strip shape multivariable control problem," in Proc. IASTED Symp. Appl. Contr. Identification, Copenhagen, 1983.

[10] , "Shape control in Sendzimir mills using both crown and intermediate roll actuators," IEEE Trans. Automat. Contr., vol. AC-35, 1990.

[11] B. Noble, Applied Linear Algebra. englewood Cliffs, NJ: PrenticeHall, 1969.

[12] J. V. Ringwood, "Multivariable control using the singular value decomposition in steel rolling with quantitative robustness assessment," Control Eng. Practice, vol. 3, no. 4, 1995.

[13] T. Sheppard, "Shape in metal strip: The state of the art," in Proc. Metals Soc. Conf. on Shape Control, Chester, U.K., 1976, pp. 11-18.

[14] O. G. Sivilotti and B. Johansson, "Measurement and control of strip flatness in cold rolling," in IEEE Conf. Record, 1969, pp. 251-259.

[15] L. Jiachuang, "Analysis of profile and shape control in flat rolling," in Int. Conf. Steel Rolling-Science and Tech. of Flat Rolled Products, Tokyo, 1980, pp. 713-724.

[16] D. G. Bates, J. V. Ringwood, and A. M. Holohan, "Robust shape control in a Sendzimir cold-rolling steel mill," in Proc. 13th IFAC World Congress, San Francisco, 1996.

[17] J. V. Ringwood, "Least squares identification with application to shape control in a Sendzimir mill," in Proc. American Control Conf. (ACC'85), Boston, MA, June 1985

John V. Ringwood (M'87-SM'97) received the Diploma degree in electrical engineering from Dublin Institute of Technology, Dublin, Ireland, and the Ph.D. degree in control systems from Strathclyde University, U.K.

He has been with the School of Electronic Engineering at Dublin City University since 1985 and is currently a Senior Lecturer there. From 1991 to 1992 , he was a Visiting Research Fellow at Massey University, New Zealand. He has acted as consultant to a number of comapnies, including Moog Ltd., Westinghouse, ESB, Anchor Products, and Valley Power. His interests include time series modeling, application of black box modeling and control techniques, modeling using intelligent techniques and computer-based learning.

Dr. Ringwood is a Chartered Engineer and a Member of the Institute of Electrical Engineers. 